\title{
Rat neonatal beta cells lack the specialised metabolic phenotype of mature beta cells
}

\author{
A. Jermendy • E. Toschi $\cdot$ T. Aye $\cdot$ A. Koh • \\ C. Aguayo-Mazzucato • A. Sharma • G. C. Weir • \\ D. Sgroi $\cdot$ S. Bonner-Weir
}

Received: 31 August 2010 / Accepted: 25 November 2010 /Published online: 16 January 2011

(C) Springer-Verlag 2011

\begin{abstract}
Aims/hypothesis Fetal and neonatal beta cells have poor glucose-induced insulin secretion and only gain robust glucose responsiveness several weeks after birth. We hypothesise that this unresponsiveness is due to a generalised immaturity of the metabolic pathways normally found in beta cells rather than to a specific defect.

Methods Using laser-capture microdissection we excised beta cell-enriched cores of pancreatic islets from day 1 (P1) neonatal and young adult Sprague-Dawley rats in order to compare their gene-expression profiles using Affymetrix U34A microarrays (neonatal, $n=4$; adult, $n=3$ ).

Results Using dChip software for analysis, 217 probe sets for genes/38 expressed sequence tags (ESTs) were signif-
\end{abstract}

A. Jermendy and E. Toschi contributed equally to this study.

Electronic supplementary material The online version of this article (doi:10.1007/s00125-010-2036-x) contains supplementary material, which is available to authorised users.

A. Jermendy $\cdot$ E. Toschi $\cdot$ T. Aye $\cdot$ A. Koh $\cdot$

C. Aguayo-Mazzucato $\cdot$ A. Sharma $\cdot$ G. C. Weir $\cdot$

S. Bonner-Weir $(\square)$

Section on Islet Cell and Regenerative Biology,

Research Division, Joslin Diabetes Center,

Department of Medicine, Harvard Medical School,

One Joslin Place,

Boston, MA 02215, USA

e-mail: Susan.Bonner-Weir@joslin.harvard.edu

A. Jermendy

1st Department of Pediatrics, Semmelweis University,

Budapest, Hungary

D. Sgroi

Molecular Pathology Unit, Massachusetts General Hospital, Department of Pathology, Harvard Medical School,

Boston, MA, USA icantly higher and 345 probe sets for genes/33 ESTs significantly lower in beta cell-enriched cores of neonatal islets compared with those of adult islets. Among the genes lower in the neonatal beta cells were key metabolic genes including mitochondrial shuttles (malate dehydrogenase, glycerol-3-phosphate dehydrogenase and glutamate oxalacetate transaminase), pyruvate carboxylase and carnitine palmitoyl transferase 2. Differential expression of these enzyme genes was confirmed by quantitative PCR on RNA from isolated neonatal (P2 until P28) and adult islets and with immunostaining of pancreas. Even by 28 days of age some of these genes were still expressed at lower levels than in adults.

Conclusions/interpretation The lack of glucose responsiveness in neonatal islets is likely to be due to a generalised immaturity of the metabolic specialisation of pancreatic beta cells.

Keywords Glucose responsiveness · Immature function . Mitochondrial shuttles $\cdot$ Neonatal islet

$\begin{array}{ll}\text { Abbreviations } \\ \text { DAVID } & \begin{array}{l}\text { Database for Annotation, Visualization } \\ \text { and Integrated Discovery }\end{array} \\ \text { dChip } & \text { DNA-Chip Analyzer } \\ \text { EST } & \begin{array}{l}\text { Expressed sequence tag } \\ \text { GSIS }\end{array} \\ \text { Glucose-stimulated insulin secretion } \\ \text { LCB } & \text { Lower confidence boundary } \\ \text { LCM } & \text { Laser capture microdissection } \\ \text { PK } & \text { Pyruvate kinase } \\ \text { qPCR } & \text { Quantitative real-time PCR } \\ \text { SLC27A1/5 } & \text { Solute carrier family 27 (fatty acid } \\ & \text { transporter), member 1/5 }\end{array}$




\section{Introduction}

Beta cells are highly differentiated cells that maintain blood glucose levels in the physiologically relevant range by rapid and precise changes in insulin secretion in response to nutrient secretagogues, particularly glucose [1]. Specialised metabolic pathways ensure the coupling of external nutrient stimuli to insulin secretion in adult beta cells [2]. During glycolysis in the adult beta cell, glucose is metabolised to pyruvate and the glucose-derived carbons are then oxidised in the mitochondria [3]. The very low expression of lactate dehydrogenase and monocarboxylate transporters results in negligible lactate production [4]. Glycolysis-derived cytosolic NADH is reoxidised in mitochondrial membrane shuttles, which include glycerol phosphate, and the malate-aspartate, pyruvate-citrate and pyruvate-malate shuttles $[5,6]$. In addition, beta cells show strong pyruvate carboxylase activity [6] that facilitates the flux of pyruvate into the pyruvate-malate and pyruvate-citrate shuttles. Glucose oxidation promotes the production of ATP, the key metabolic coupling factor for glucose-stimulated insulin secretion (GSIS). The closing of ATP-dependent $\mathrm{K}^{+}$channels results in depolarisation, opening of voltage-activated $\mathrm{Ca}^{2+}$ channels and subsequent exocytosis of the insulin granules. GSIS can also be stimulated by glucose metabolism, which exerts its effect independently of the ATP-dependent $\mathrm{K}^{+}$channels, working through as yet undefined mechanisms [7]. In addition, there are other metabolic and neurohormonal amplifying pathways in beta cells that can potentiate insulin release.

Although the metabolism of adult beta cells has been characterised extensively, that of fetal/neonatal beta cells is less well understood. Both human and rodent fetal and rodent neonatal islets [8-12] lack glucose responsiveness despite adequate insulin reserves [13], but secrete insulin in response to amino acids, particularly leucine $[14,15]$. When newborn (P1) Sprague-Dawley rat islets were tested in a dose-response experiment using different secretagogues, insulin secretion was impaired in response to all stimuli; insulin secretion in response to different secretagogues started to rise at 1 week of age $[15,16]$. The insulin secretion in response to glucose of perifused islets of different-aged rats [17] was small compared with adult islets at P7, with a slight increment at P14 and a doubling at $\mathrm{P} 21$; however, even at P21, the response was less than half of that of adult islets. A variety of mechanisms focusing on individual genes have been proposed to account for immature insulin secretion, including lack of inhibition of fatty acid oxidation by glucose metabolism [17], lack of increased ATP content in response to glucose stimulation [18], and low levels and activity of mitochondrial glycerol3-phosphate dehydrogenase and mitochondrial malate dehydrogenase, both NADH shuttle enzymes [19].
Postulating that the mechanisms responsible of immaturity are more complex, we used microarray analysis to compare the gene expression profile of neonatal (P1) and adult beta cells excised by laser-capture microdissection. Key metabolic enzymes characteristic of the beta cell phenotype were among the genes with differentially low expression in neonatal beta cells. These findings were confirmed with quantitative realtime PCR (qPCR) and immunostaining. Moreover, during the first weeks of postnatal life, expression of these genes increased in islets as insulin secretory responsiveness matured. Thus, the generalised low expression of key metabolic enzymes, particularly the mitochondrial shuttles, may account for the poor glucose-responsiveness of neonatal beta cells.

\section{Methods}

Animals Adult Sprague-Dawley rats - male, female pregnant and female with P1 litters - were purchased (Taconic Farms, Germantown, NY, USA). Pregnant animals were checked until delivery in order to have $\mathrm{P} 1$ animals ( $\mathrm{P} 0$ as day of birth). Animals were kept under conventional conditions in climatised rooms, with free access to tap water and standard pelleted food. All procedures were approved by the Joslin Institutional Animal Care and Use Committee. Neonatal pups were nursed until they were killed at P1, P2, P7, P9, P11, P13, P15, P21 or P28. For immunostaining, excised pancreases $(n=3$ for P2, P7 and adult) were fixed by immersion in $4 \%$ paraformaldehyde, and stored in PBS until paraffin embedding and sectioning. For laser capture microdissection (LCM), excised pancreas in TissueTek OCT medium (VWR Scientific Products, San Diego, CA, USA) was rapidly frozen in chilled isobutane and immediately stored at $-80^{\circ} \mathrm{C}$.

Adult male rats were anaesthetised with Nembutal for islet isolation by collagenase digestion [20] with rodent Liberase RI (Roche, Indianapolis, IN, USA). Islets from two adults were pooled as one sample. For neonatal islets, rats were decapitated and pancreases from a litter (10-12 pups) were pooled, finely minced and digested with Liberase solution. Adult and neonatal islets were purified by gradient separation using Histopaque-1077 (Sigma, St Louis, MO, USA) and after overnight culture were handpicked under a stereomicroscope to ensure high purity. Islets were put in Trizol reagent (Invitrogen, Carlsbad, CA, USA) for RNA extraction and stored at $-80^{\circ} \mathrm{C}$.

Laser-capture microdissection Frozen sections $(8 \mu \mathrm{m})$ on uncoated slides were quickly processed for LCM: first in $70 \%$ ethanol for $10 \mathrm{~s}$, haematoxylin for $40 \mathrm{~s}$, dehydrated for $10 \mathrm{~s}$ each in $70 \%$ and $95 \%$ ethanol, stained with eosin for $10 \mathrm{~s}$ and finally dehydrated for $3 \mathrm{~min}$ in $100 \%$ ethanol followed by xylene for a minimum of $2 \mathrm{~min}$. Once sections were air-dried, beta cell-enriched cores of islets were 
microdissected using a PixCell II LCM system (Arcturus, Mountain View, CA, USA). For each sample, at least 4,000 'hits' from two to five islets per section were excised from 10-20 sections. Total RNA from each sample was extracted using a modification of the RNA microisolation protocol as described [21-23]. T7-based RNA amplification was carried out using the RiboAmp kit (Arcturus) according to the manufacturer's recommendations. To obtain enough amplified RNA for microarray, a second round of RNA amplification was performed [24].

Microarray hybridisation Biotinylated cDNA (BioArray HighYield RNA Transcript Labeling kit; Enzo Life Sciences, Farmingdale, NY, USA) of four adult and four neonatal beta cell-enriched samples were run on Affymetrix GeneChip Rat Genome U34A (Affymetrix, Santa Clara, CA, USA; adult by MGH Cancer Center DNA Microarray Core Facility; neonatal by Joslin DERC Genomic Core). Data have been deposited in a MIAME-compliant database (GEO series accession number GSE24790).

Analysis was performed using a DNA-Chip Analyzer (dChip; Harvard School of Public Health, Boston, MA, USA; www.dchip.org). This software uses the model-based expression analysis, which allows probe-level analysis on multiple arrays. As the LCM RNA samples were amplified before labelling, we followed the manufacturer's recommendation of using 50\% masked data for analysis. Principal component analysis for sample classification with dChip software revealed that one of the adult samples was an outlier, therefore this array was excluded from further analysis. Data from multiple arrays are normalised to a common baseline array having the median overall brightness. Thus, by pooling information across multiple arrays, it is possible to assess the standard errors for the expression indices and to calculate confidence intervals for fold changes. Lower confidence boundary (LCB) and $p$ values $(<0.050)$ were used to assess differentially expressed genes. Differentially expressed genes were classified into functionally related clusters using Database for Annotation, Visualization and Integrated Discovery (DAVID) software $[25,26]$. The functional annotation tool of DAVID using high-classification stringency identified enriched functionally related gene groups; the enrichment $p$ values are reported.

$R N A$ extraction from isolated islets and reverse transcription After quantification by spectrophotometry, $500 \mathrm{ng}$ total RNA from each islet sample was used as starting material for cDNA. Reverse transcription was done in $25 \mu$ l reaction solution using Superscript II reverse transcriptase (Invitrogen) according to the manufacturer's recommendations.

Quantitative real-time PCR ( $q P C R$ ) for confirmation of differential expression of genes qPCR with SYBR Green detection was performed using the ABI7300 Real-time PCR System (Applied Biosystems, Foster City, CA, USA) with primers (see Electronic supplementary material [ESM] Table 1) designed using Primer Express (Applied Biosystems). Each primer set displayed approximately equal efficiency for amplification of target cDNA. The reactions were set by mixing $10 \mu \mathrm{l}$ SYBR Green Master Mix (Applied Biosystems) with $1 \mu \mathrm{l}$ of each $5^{\prime}$ and $3^{\prime}$ oligonucleotides $(10 \mathrm{pmol} / \mu \mathrm{l})$ and $1 \mu \mathrm{l}$ cDNA sample $(10 \mathrm{ng} / \mu \mathrm{l})$. After normalisation of the gene of interest to a control ribosomal gene (L32) [27], the comparative $C_{t}$ (threshold cycle) method was used to calculate relative gene expression levels. Neonatal islet mRNA levels reported as mean \pm SEM were calculated relative to adult levels [28].

Immunostaining Paraffin sections were blocked for endogenous peroxidase, microwaved in $10 \mathrm{mmol} / \mathrm{l}$ citrate buffer $\mathrm{pH} 6.0$ for $15 \mathrm{~min}$ at $20 \%$ power and then incubated overnight at $4^{\circ} \mathrm{C}$ with anti-pyruvate kinase antibody $(1: 100$ goat-anti rabbit, US Biologicals, Swampscott, MA, USA), then with biotinylated anti-goat $\operatorname{IgG}$ (Vector Laboratories) $1 \mathrm{~h}$, with $\mathrm{ABC}$ reagent $1 \mathrm{~h}$ and visualised with VIP (Vector VIP substrate kit for peroxidase, Vector Labs, Burlingame, CA, USA). Incubations with anti-rabbit glycerol-3-phosphate dehydrogenase (1:100, the kind gift of M. MacDonald, Department of Pediatrics, University of Wisconsin, WI, USA), donkey biotinylated anti-rabbit IgG (1:400), were followed by streptavidin-conjugated Alexafluor Green (1:400). Sections were double stained for insulin (guinea pig anti-human, 1:200, Linco Research, St Charles, MO, USA) with Texas Redconjugated Affinipure donkey anti-guinea pig $\operatorname{IgG}(1: 400)$ as secondary antibody. Images were taken with an Olympus BH2 or, in confocal mode, a Zeiss 410 or 710 LSM microscope. Sections of different ages were stained and photographed in parallel using the same settings, so the relative intensities reflect the protein levels. For beta cell composition, pancreatic sections double stained with anti-insulin and a cocktail of anti-non-beta cell hormones were imaged by tile scan collection, and then the insulin-positive area of all clusters at least $35 \mu \mathrm{m}$ diameter were quantified as proportion of total islet area (ESM Table 2).

Data analysis For statistical analysis, unpaired Student's $t$ test was used. To see differences among groups, ANOVA was used followed by post hoc analysis (Tukey's). A $p$ value $<0.050$ was considered statistically significant.

\section{Results}

Microarray analysis revealed different mRNA expression patterns in neonatal compared with adult beta cells Using 
dChip analysis on $50 \%$ masked probes and the highstringency LCB cutoff of 2 and $p<0.050$, we found 217 genes $/ 38$ expressed sequence tags (ESTs) higher in neonatal compared with adult beta cell-enriched cores and 345 genes/33 ESTs lower in neonatal; with a false-discovery rate of $4.9 \%$. Hierarchical clustering analysis of differentially expressed genes showed a clear separation between neonatal and adult control samples (Fig. 1). Differentially expressed genes ranged widely across biochemical functions, including metabolism, cell cycle, ion channels and cell surface proteins (ESM Tables 3 and 4).

For an unbiased analysis of the differentially expressed genes in neonatal beta cells (LCB $>2, p<0.050$ ), DAVID functional annotation clustering $[25,26]$ was used. The top three enriched clusters were extracellular matrix, ribosome and the developmental process as might be expected for developing tissue (ESM Fig. 1a). With the differentially lower genes, the three most enriched functional clusters were organelle envelope/mitochondrial membrane, organelle lumen and negative regulation of apoptosis (ESM Fig. 1b). The enrichment score of the organelle/mitochondrial membrane genes (11.92, which is more than double that of other categories) indicates a very strong over-representation. This robust enrichment suggests a systemic lower expression of mitochondrial genes, especially those for the membranebound proteins and shuttle enzymes.

Less stringent criteria (LCB cutoff of 1.2 and $p<0.050$ ) were used to selectively analyse genes important in the beta cell phenotype. The number of genes higher in neonatal samples increased to 363 probe sets for genes/54 ESTs and those with reduced gene expression increased to 685 genes/ 68 ESTs. It is striking that a high percentage of genes in the mitochondrial shuttles and fatty acid metabolism were differentially expressed. We focused on differentially expressed genes of the beta cell-specialised metabolic pathway, grouping them into functional clusters (Table 1 and ESM Table 5).
Beta cell monitoring of circulating glucose concentrations is made possible by the co-expression of GLUT2 glucose transporter and high $K_{\mathrm{m}}$ glucokinase [29]; neither of these genes was differentially expressed. However, hexokinase 2 , which is expressed at very low levels in functional adult beta cells [30], was more highly expressed in neonatal beta cells $(p=0.046)$. Gene expression of other glycolytic enzymes was unchanged (Table 1) except two genes that were lower in neonatal beta cells: phosphoglycerate kinase $(p=0.042)$ and phosphofructokinase $\mathrm{L}$ and $\mathrm{M}$ types, which have been implicated in oscillatory insulin release [31], $(p=0.024$ and 0.006 , respectively). Lactate dehydrogenase A and monocarboxylate transporter 1 (also known as Slc16al), minimally expressed in adult beta cells [30], were present at higher levels in neonatal samples, which however did not reach statistical significance in part because of variability.

Glucose is metabolised to pyruvate, and pyruvate provides the carbon skeleton to the Krebs cycle. Interestingly, while genes of the pyruvate dehydrogenase enzyme complex were not differentially expressed, pyruvate carboxylase $(P c)$, the key anaplerotic enzyme in mitochondria, was markedly lower in neonatal beta cells $(p=0.004)$. The anaplerotic mechanism of pyruvate and pyruvate cycling is closely correlated with GSIS in beta cells $[5,6,32,33]$. It was striking that a number of mitochondrial shuttle enzymes, so key to the efficient generation of ATP and pyruvate cycling in the adult beta cell, had less than $20 \%$ of adult expression in neonatal beta cells (Table 1). Shuttle genes with lower neonatal expression included malate dehydrogenase 1 (Mdh1; 15-fold lower, $p=0.020)$ and $2(M d h 2 ; p=0.027)$, aspartate aminotransferase 1 (Got $1 ; p=0.001)$ and 2 (Got2; $p=0.016$ ), glycerol-3-phosphate dehydrogenase 1 ( $G p d 1 ; p=$ $0.016)$ and $2(G p d 2 ; p=0.015)$. Other pyruvate-shuttling genes with neonatally lower expression included Slc25al (mitochondrial citrate/isocitrate carrier; $p=0.038$ ) and cytosolic malic enzyme (Mel), a critical component of pyruvate-
Fig. 1 Heat map display of the differentially expressed genes in laser-captured enriched beta cells from neonatal (P1) and adult pancreases. Using dChip analysis with LCB cutoff of 2 and $p<0.050$, adult beta cells had 345 genes and 33 ESTs more highly expressed than in neonates, while neonatal beta cells had 217 genes and 38 ESTs more highly expressed than in adult

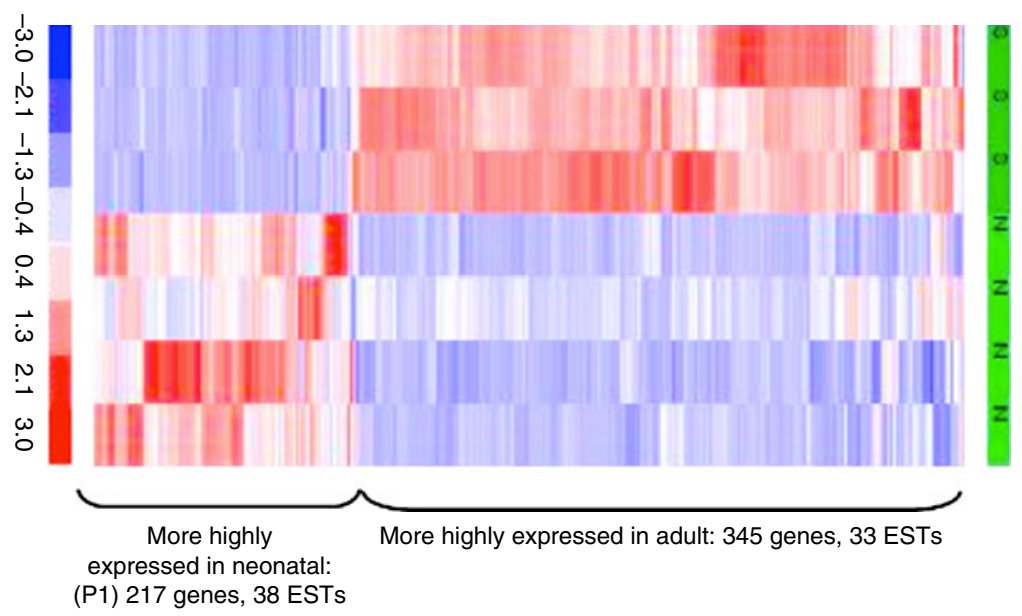

Adult - control 1

Adult - control 2

Adult - control 3

Neonatal 1

Neonatal 2

Neonatal 3

Neonatal 4

Neonatal 4 
Table 1 Expression of metabolic genes in beta cells grouped into functional categories (glycolysis, Krebs cycle, mitochondrial shuttles)

\begin{tabular}{|c|c|c|c|c|c|c|}
\hline Probe set & Gene symbol & Adult & Neonate & $\mathrm{FC}$ & LCB & $p$ value \\
\hline \multicolumn{7}{|l|}{ Glycolysis } \\
\hline L28135_at & Slc2a2 (Glut2) & $16,679 \pm 1,942$ & $7,253 \pm 3,157$ & -2.30 & -1.28 & 0.055 \\
\hline D63834_at & Slc16a1 (Mct1) & $49 \pm 17$ & $286 \pm 79$ & 5.84 & 2.74 & 0.055 \\
\hline rc_AI012593_at & $H x k 1$ & $1,535 \pm 427$ & $4,245 \pm 1,415$ & 2.76 & 1.16 & 0.150 \\
\hline M68971_at & $H x k 2^{*}$ & $1,169 \pm 2$ & $3278 \pm 642$ & 2.80 & 1.90 & 0.046 \\
\hline X53588_at & Gck $(H x k 4)$ & $1,751 \pm 172$ & $1,847 \pm 519$ & 1.05 & 0.56 & 0.871 \\
\hline X58865mRNA_at & $P f k l^{*}$ & $1,290 \pm 157$ & $538 \pm 74$ & -2.40 & -1.77 & 0.024 \\
\hline U25651_at & Pfkm ${ }^{*}$ & $6,665 \pm 228$ & $3,140 \pm 601$ & -2.12 & -1.60 & 0.006 \\
\hline L25387_at & Pfkp & $99 \pm 61$ & $144 \pm 71$ & 1.45 & 0.24 & 0.653 \\
\hline M12919mRNA\#2_at & Aldoa & $96 \pm 38$ & $488 \pm 245$ & 5.07 & 0.85 & 0.207 \\
\hline X02284_at & Aldob & $524 \pm 42$ & $481 \pm 66$ & -1.09 & -0.85 & 0.604 \\
\hline X06984cds_s_at & Aldoc & $277 \pm 129$ & $1,416 \pm 731$ & 5.11 & 0.73 & 0.218 \\
\hline rc_AI104399_at & Tpil & $6,406 \pm 1,864$ & $3,446 \pm 1,261$ & -1.86 & -0.84 & 0.263 \\
\hline M17701_s_at & Gapdh & $800 \pm 142$ & $320 \pm 113$ & -2.49 & -1.40 & 0.055 \\
\hline rc_AA892797_at & $P g k 1^{*}$ & $2,088 \pm 276$ & $900 \pm 341$ & -2.32 & -1.34 & 0.042 \\
\hline S63233_at & Pgam1 & $4,582 \pm 574$ & $3,728 \pm 695$ & -1.23 & -0.86 & 0.387 \\
\hline Z17319_at & Pgam2 & $306 \pm 69$ & $3,505 \pm 1,440$ & 11.46 & 3.60 & 0.113 \\
\hline X02610_at & Enol & $160 \pm 49$ & $287 \pm 145$ & 1.80 & 0.30 & 0.457 \\
\hline X07729exon\#5_s_at & Eno2 & $2,034 \pm 28$ & $1187 \pm 615$ & -1.71 & -0.92 & 0.262 \\
\hline rc_AA851223_at & Eno3 & $1,036 \pm 307$ & $61 \pm 47$ & -16.86 & -5.83 & 0.083 \\
\hline X05684_at & Pklr & $1,871 \pm 624$ & $363 \pm 127$ & -5.16 & -2.08 & 0.132 \\
\hline rc_AA818951_at & Pkm2 & $40,625 \pm 2,773$ & $59,823 \pm 17,780$ & 1.47 & 0.75 & 0.361 \\
\hline M54926_at & Ldha & $3,265 \pm 865$ & $20,975 \pm 9,144$ & 6.42 & 1.75 & 0.148 \\
\hline \multicolumn{7}{|l|}{ Krebs cycle } \\
\hline rc_AA799598_at & Pdhal & $2,536 \pm 193$ & $1,487 \pm 393$ & -1.71 & -1.16 & 0.071 \\
\hline Z18878cds_at & Pdha2 & $79 \pm 48$ & $93 \pm 66$ & 1.17 & 0.00 & 0.876 \\
\hline rc_AA892828_at & $P d h b$ & $4,343 \pm 713$ & $1,945 \pm 649$ & -2.23 & -1.30 & 0.060 \\
\hline D10655_at & Dlat & $6,328 \pm 1,563$ & $4,950 \pm 1,480$ & -1.28 & -0.66 & 0.552 \\
\hline L22294_at & $P d k 1$ & $1,600 \pm 385$ & $154 \pm 47$ & -10.38 & -5.45 & 0.062 \\
\hline U10357_g_at & $P d k 2^{*}$ & $1,955 \pm 379$ & $222 \pm 91$ & -8.80 & -4.61 & 0.038 \\
\hline AF034577_at & $P d k 4$ & $39 \pm 8$ & $34 \pm 9$ & -1.14 & -0.68 & 0.699 \\
\hline U32314_g_at & $P c^{*}$ & $8,758 \pm 940$ & $1,431 \pm 654$ & -6.12 & -3.37 & 0.004 \\
\hline rc_AI638990_at & Aco 2 & $221 \pm 88$ & $6,294 \pm 3764$ & 28.53 & 0.46 & 0.205 \\
\hline rc_AA892314_at & $\operatorname{Idh} 1$ & $3,938 \pm 237$ & $9,800 \pm 1,925$ & 2.49 & 1.67 & 0.055 \\
\hline rc_AA892808_at & $I d h 3 g$ & $2,024 \pm 83$ & $2,702 \pm 293$ & 1.34 & 1.09 & 0.100 \\
\hline D90401_at & Dlst & $161 \pm 69$ & $161 \pm 64$ & -1.00 & -0.27 & 0.999 \\
\hline J03621_at & Suclg $1^{*}$ & $1,796 \pm 268$ & $724 \pm 170$ & -2.48 & -1.61 & 0.033 \\
\hline rc_AA800250_at & Sdha & $5,729 \pm 1,571$ & $4,941 \pm 570$ & -1.16 & -0.62 & 0.675 \\
\hline J04473_at & $F h 1$ & $11,073 \pm 1,611$ & $6,447 \pm 1,837$ & -1.72 & -1.07 & 0.117 \\
\hline rc_AI010480_at & $M d h 2^{*}$ & $2,727 \pm 467$ & $712 \pm 293$ & -3.83 & -2.07 & 0.027 \\
\hline \multicolumn{7}{|l|}{ Mitochondrial shuttles } \\
\hline AB002558_at & $G p d 1^{*}$ & $263 \pm 20$ & $142 \pm 27$ & -1.86 & -1.37 & 0.016 \\
\hline U83880UTR\#1_g_at & $G p d 2^{*}$ & $9,746 \pm 1,415$ & $2,508 \pm 1,187$ & -3.89 & -2.04 & 0.015 \\
\hline AF093773_s_at & $M d h 1^{*}$ & $65,299 \pm 9,280$ & $4,245 \pm 1,499$ & -15.38 & -9.03 & 0.020 \\
\hline rc_AI010480_at & $\operatorname{Mdh} 2^{*}$ & $2,727 \pm 467$ & $712 \pm 293$ & -3.83 & -2.07 & 0.027 \\
\hline J04171_at & Got1 $^{*}$ & $7,296 \pm 439$ & $1,341 \pm 246$ & -5.44 & -4.09 & 0.001 \\
\hline M18467_at & 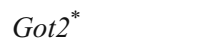 & $6,162 \pm 417$ & $4,096 \pm 323$ & -1.50 & -1.27 & 0.016 \\
\hline rc_AI008020_at & Mel & $10,982 \pm 2,349$ & $1,255 \pm 328$ & -8.75 & -5.00 & 0.051 \\
\hline J05210_at & Acly & $6,679 \pm 824$ & $4,700 \pm 1,741$ & -1.42 & -0.84 & 0.360 \\
\hline
\end{tabular}


Table 1 (continued)

\begin{tabular}{|c|c|c|c|c|c|c|}
\hline Probe set & Gene symbol & Adult & Neonate & $\mathrm{FC}$ & LCB & $p$ value \\
\hline L12016_at & $\operatorname{Slc} 25 a 1(\mathrm{Cic})^{*}$ & $2,674 \pm 375$ & $1,128 \pm 154$ & -2.37 & -1.70 & 0.038 \\
\hline AJ223355_at & Slc25a10 (Dic) & $185 \pm 17$ & $210 \pm 47$ & 1.13 & 0.70 & 0.646 \\
\hline
\end{tabular}

Microarray signals of adult ( $n=3)$ and neonatal $(n=4)$ samples. Genes of the various pathways are listed with the mean $\pm \mathrm{SEM}$, fold change (FC), LCB and $p$ value as determined with dChip

Where multiple probe sets were on the microarray, the more stringent_at set is listed; if no_at probe set, the set with highest expression is listed

${ }^{*}$ Significance $(p<0.050$, fold change of 1.5 or greater, LCB of 1.2 , and signal mean difference of at least 100$)$

malate shuttle, which had almost ninefold difference in expression but just missed statistical significance $(p=0.051)$.

Notably, gene expression levels of Krebs cycle enzymes were not statistically different between adult and neonatal beta cells, except for succinate-CoA ligase 1 (also known as succinyl-CoA synthetase; Suclg1) and Mdh2, which is also a key enzyme of the pyruvate-malate shuttle (Table 1).

Of the 53 genes present on the microarray encoding subunits of the electron transport chain complexes I-IV and the ATP synthase, ten were differentially lower in neonatal beta cells $(p<0.050$; ESM Table 5$)$. Only $N d 3$, the only mitochondrial DNA-encoded electron transport chain gene on the microarray, and Cox8a were more highly expressed in the neonatal beta cells ( $p=0.029$ and 0.042 , respectively).

With regard to lipid metabolism genes, fatty acid synthase was significantly upregulated in neonatal beta cells $(p=0.009)$. Carnitine palmitoyl transferase $1(C p t l a)$, which transfers acyl groups from the cytosol to the mitochondria for beta-oxidation, was also upregulated whereas the inner mitochondrial membrane Cpt2 was eightfold lower in neonatal beta cells ( $p=0.046$ and 0.021 , respectively). There is a switch in fatty acid transporters with Slc27a5, being four to five times higher in the neonate beta cell $(p=0.032)$, and Slc27al, eight times higher in the adult $(p=0.001)$. Expression levels of genes encoding mitochondrial enzymes of beta-oxidation were largely unchanged, except for certain subunits of the hydroxyacylCoA dehydrogenase complex (ESM Table 5).

For genes implicated in insulin exocytosis, very few expression differences were found. Synaptotagmin 5, Vamp2 and Stxbp1 had lower expression in neonatal beta cells compared with adult $(p=0.002,0.041$, and 0.002 , respectively); while synaptotagmin 6 and 8 were higher ( $p=$ 0.017 and 0.011 , respectively). However, genes encoding key SNARE proteins of the exocytotic machinery Snap 25 and syntaxin 1A (Stxla) did not differ. Similarly, those encoding ATP-sensitive $\mathrm{K}^{+}$-channel subunits Kir6.2 (also known as Kcnj11), Sur1 (also known as $A b c c 8$ ), delayed rectifier $\mathrm{K}^{+}$channel $K v 2.1$ (also known as Kcnb1) and voltage-dependent $\mathrm{Ca}^{2+}$-channels Cav1.2 (also known as Cacnalc) and Cav1.3 (also known as Cacnald) did not differ. It is noteworthy that insulin 2 gene expression was almost identical in neonatal and adult beta cells as well.

Confirmation of microarray data with $q P C R$ Differential expression seen in microarray data (Fig. 2) was confirmed with qPCR on RNA from isolated neonatal P2, P7 and adult islets. The percentage of beta cells/islets did not differ significantly among P2, P21 and adult islets; only at P11 was it significantly lower (20\%) than adult (ESM Table 2). Even so, neonatal islets expressed only $10-20 \%$ of adult levels of the metabolic genes: Pc, Me1, Mdh1, Got1, Gpd2 and Cpt2. In contrast, Cpt1 levels at P2 did not differ from adult but at P7 decreased to $45 \%$ of adult (Fig. 3). Metabolic genes encoding pyruvate kinase (PK) and glycerol-3-phosphate dehydrogenase 1 (GPD2) were evaluated by immunostaining; the PK antibody does not distinguish between PK muscle isoform (PKM) and PK liver and RBC isoform (PKLR) isoforms. Both enzymes had low-intensity staining in P2 islets but had strong staining in adult islets (Fig. 4). At P7, PK protein was intermediate between neonatal and adult islets. In contrast GPD2, with little to no staining at birth, was clearly produced at $\mathrm{P} 7$ with similar pattern and intensity as adult islets.

To study the development of the characteristic metabolic profile of adult beta cells, we examined expression of these genes over the first postnatal month (Fig. 5). Several different expression patterns were found, perhaps reflecting different regulation. $M e 1$ and $P c$ had low expression even at P7 but with sharp increases at P9; $\mathrm{Mel}$ had another sharp increase at $\mathrm{P} 28$ while $P c$ gradually increased from P9 onwards (Fig. 5a). In contrast, Mdh1, Got1 and Gpd2 (Fig. 5b) had stable low expression with a moderate increase only at P28. While Cpt2 expression followed a pattern similar to that of Mdh1, Got1 and Gpd2, Cpt1 decreased further to $20 \%$ of adult by P9 (Fig. 5c). For $P k$ (primers selective for $P l k r$ ) there was a sharp increase at $\mathrm{P} 9$, reaching levels that were highly variable but consistently high as or higher than adult through P28 (Fig. 5d). 

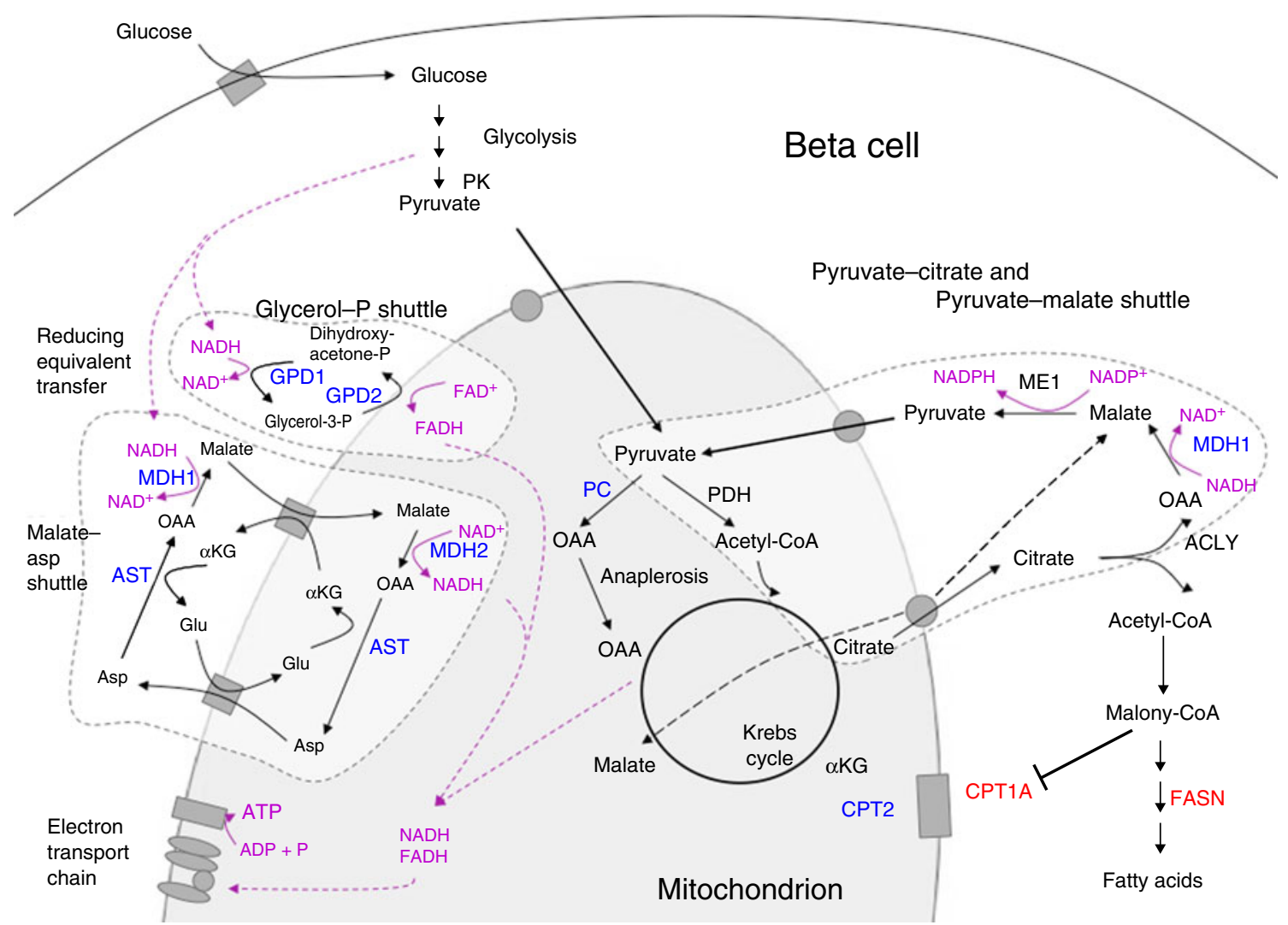

Fig. 2 Metabolic pathways involved in glucose-stimulated insulin secretion in beta cells. Based on dChip analysis of microarray data of enriched beta cell samples from neonatal vs adult samples, genes with lower expression in the neonates (adult/neonatal $\leq-1.2$ ) are in blue and those with higher expression in neonates (neonatal/adult $\geq 1.2$ ) are in red; unchanged in black. Asp, aspartate; AST, aspartate aminotransferase; ACLY, ATP citrate lyase; CPT1A, carnitine palmitoyl

\section{Discussion}

The physiological experiments of Grill et al. [16] and Bliss et al. [17] showed a continuum in maturation of insulin secretion from birth to adult in response to glucose and to different secretagogues [16]. In both studies glucosestimulated insulin secretion was minimal at P7 and even at 3 weeks age it was not as robust as in the adult islets. To address the mechanisms of this immature insulin secretion we performed gene expression studies using beta cellenriched samples and microarray technology. Our microarray results were confirmed for a number of genes by qPCR analysis on isolated rat islets. While changes in the proportion of beta cells in isolated islets could have affected the results, the proportion of beta cell/islet did not significantly differ among P2, P21 and adult islets and so could not explain the gene expression results (ESM Table 2).

Comparison of the transcriptosomes of neonatal and adult beta cells showed a number of differentially expressed transferase 1; CPT2, carnitine palmitoyl transferase 2; FASN, fatty acid synthase; Glu, glutarate; GPD1, glycerol-3-phosphate dehydrogenase 1; GPD2, glycerol-3-phosphate dehydrogenase 2; MDH1, malate dehydrogenase 1; MDH2, malate dehydrogenase 2; ME1, malic enzyme 1; OAA, oxaloacetic acid; P, phosphate; PC, pyruvate carboxylase; PDH, pyruvate dehydrogenase; PK, pyruvate kinase; $\alpha \mathrm{KG}, \alpha$-ketoglutarate

genes, particularly those critical to the specialised beta cell metabolism. Pyruvate enters the Krebs cycle via decarboxylation by the pyruvate dehydrogenase complex or via

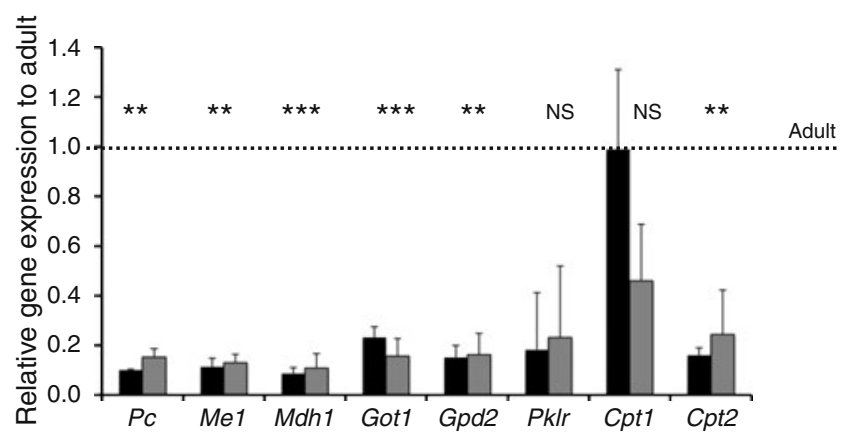

Fig. 3 qPCR confirmation of selected metabolic genes. The differential expression of selected metabolic genes was confirmed by qPCR in islets isolated from neonatal P2 (black bars, $n=4$ samples of islets pooled from a litter) and P7 rats (grey bars, $n=3$ samples of islets pooled from a litter); data are expressed relative to isolated adult islets (dotted line, $n=5$ ). One-way ANOVA with Tukey post hoc analysis. ** $p<0.01 ; * * * p<0.001$ 
Fig. 4 Immunostaining of PK and mitochondrial glycerol-3phosphate dehydrogenase 2 (GPD2). By immunostaining, the protein levels of PK (top panels, VIP as chromagen) and GPD2 (bottom panels, insulin red, GPD2 green, overlap yellow) reflect the mRNA expression through the neonatal time period

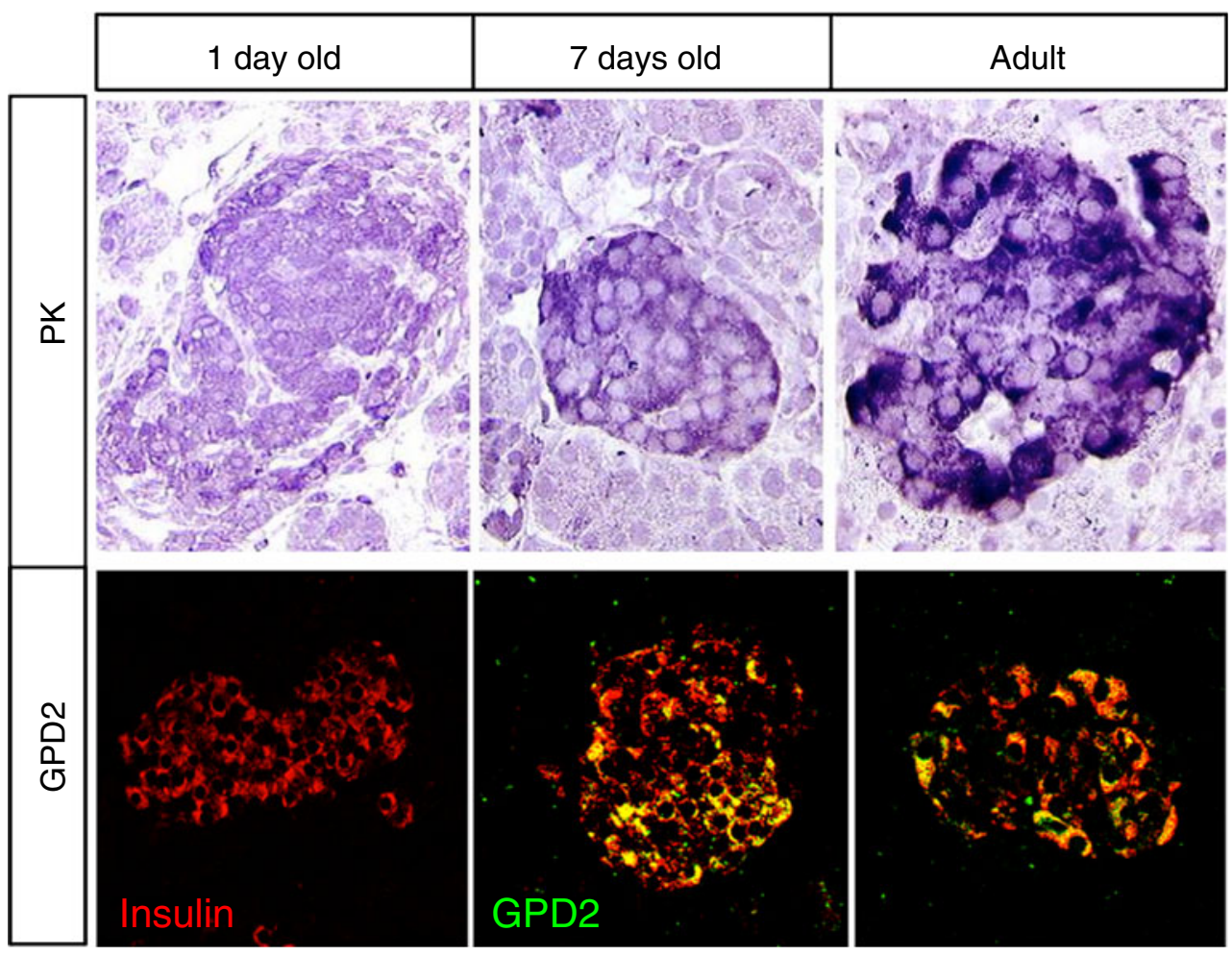

carboxylation and conversion to oxaloacetate by pyruvate carboxylase (Fig. 2). In adult rat islets approximately $50 \%$ of the glucose-derived carbon enters the Krebs cycle via carboxylation, which is correlated with glucose metabolism and insulin release [6]. Adult beta cells show strong pyruvate carboxylase activity [6] that facilitates anaplerotic flux into the Krebs cycle that leads to the generation of other putative stimulus-secretion coupling factors, such as NADPH [5, 6] or malonyl-CoA [35]. Knocking-down pyruvate carboxylase resulted in impaired glucosestimulated insulin secretion in INS-1 cells $[36,37]$ but not in isolated rat islets, in which compensatory increased enzyme activity was detected [38]. Another unique feature of the adult beta cell is the presence of mitochondrial membrane shuttles, including the glycerol phosphate, malate-aspartate, pyruvate-citrate and pyruvate-malate shuttles, through which glycolysis-derived cytosolic NADH is reoxidised $[5,6,39]$.

However, in neonatal beta cells pyruvate would be handled differently. Pyruvate carboxylase, the key anaplerotic enzyme gene, was expressed significantly lower through the third postnatal week. Both lactate dehydrogenase A (Ldha) and monocarboxylic transporter 1 (Slc16al) were elevated in the neonatal microarray data but missed significance. However, by qPCR, both were significantly elevated at P2 (seven- and fourfold; $p=0.040$ and 0.056 , respectively) and P7 (17- and 7-fold, respectively; both $p=$ 0.001 ), and by P15 LdhA was still higher than adult (9.5fold; $p=0.004$ ) but not Slc16al [40]. Their expression would divert pyruvate away from the mitochondria towards lactate production.

Both the glycerol phosphate and malate-aspartate shuttles had been reported to have lower expression in fetal rat islets compared with adult, with induced overexpression of $G p d 2$ in fetal islets improving glucose-stimulated insulin secretion [19]. Here, we show that not only the genes encoding the enzymes of the two main NADH shuttle systems (the mitochondrial Gpd2 of the glycerol phosphate shuttle, and the cytosolic Got1 and Mdh1 of the malateaspartate shuttle) had differentially lower expression in neonatal beta cells, but by qPCR so did cytosolic malic enzyme, a key enzyme in the pyruvate-malate shuttle; mRNA for all four enzymes had less than $40 \%$ expression of adults through P21 and had not reached adult levels by P28. While previous studies have shown that the impairment of any of these enzymes could lead to diminished glucose-stimulated insulin secretion in vitro $[32,37]$ or in vivo [19, 41], we found all three shuttles and pyruvate carboxylase had very low gene expression during the neonatal period. Overall, the metabolic specialisation found in adult beta cells for amplifying the ATP-derived from glycolysis is missing in neonatal beta cells.

A limitation of our study is that while we mainly investigated gene expression levels of enzymes, the activity of enzymes can be regulated at many levels. The assumption that low mRNA levels correlate with low enzymatic activity is reasonable, and our immunostaining data on PK and GPD2 proteins provide additional assurance. Both $P k$ 

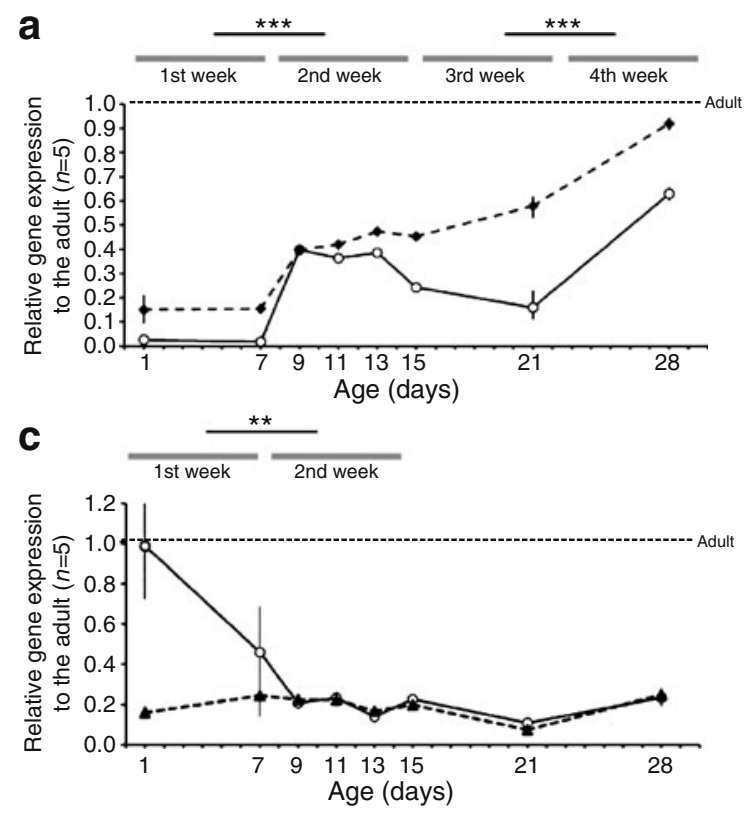

Fig. 5 Time course of expression of selected metabolic genes over the first postnatal month by $\mathrm{qPCR}$. The genes encoding the metabolic enzymes have different patterns of expression through the first postnatal month: (a) pyruvate carboxylase (black diamond), malic enzyme 1 (cytosolic) (white circle); (b) malate dehydrogenase 1 (cytosolic) (black square), glutamate oxalacetate transaminase 1 (cytosolic) (white triangle), glycerol phosphate dehydrogenase 2 (mitochondrial) (black circle) (c) carnitine palmitoyltransferase 1

and $G p d 2$ had low mRNA expression in $\mathrm{P} 2$ islets and were equally low even at 1 week after birth. However, increased protein production of both of these two enzymes at 1 week is consistent with physiological results showing a slight glucose-responsive insulin secretion in P7 islets [16].

Interestingly, $\mathrm{Cpt} 1$ and $\mathrm{Cpt} 2$, encoding carnitine palmitoyl transferases 1 and 2 , which are involved in the transport of long-chain acyl-CoA molecules from the cytosol through the mitochondrial membrane, were differentially expressed. In neonates $C p t 1$ was highly expressed while $C p t 2$ has lower expression by array data. By qPCR, Cpt1 mRNA in $\mathrm{P} 2$ neonatal islets did not differ from adult; however, Cpt2 was indeed expressed at low levels (20\%). A natural inhibitor of Cptl is the glucose-derived malonylCoA, thus fatty acid oxidation is suppressed when glucose is in abundance and oxidised in the cell. Consequently, fatty acids diverted from oxidative pathway flux into various esterified products, such as diacylglycerol and phospholipids, have been suggested as triggering factors in glucosestimulated insulin secretion. In neonatal beta cells, a possible consequence of high $\mathrm{Cpt1}$ expression could be high enzymatic activity that was not fully inhibited by malonyl-CoA, suggesting that lipids may be diverted to beta-oxidation and are potentially a more important fuel in fetal/neonatal beta cells than in adults. Interestingly, overexpression of Cpt1 has been demonstrated to diminish

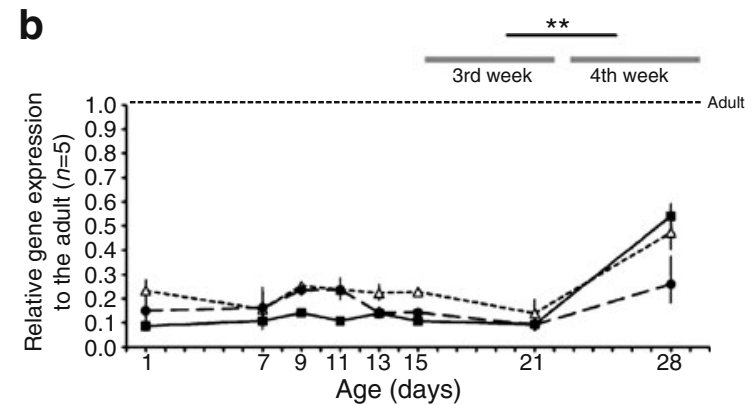

d

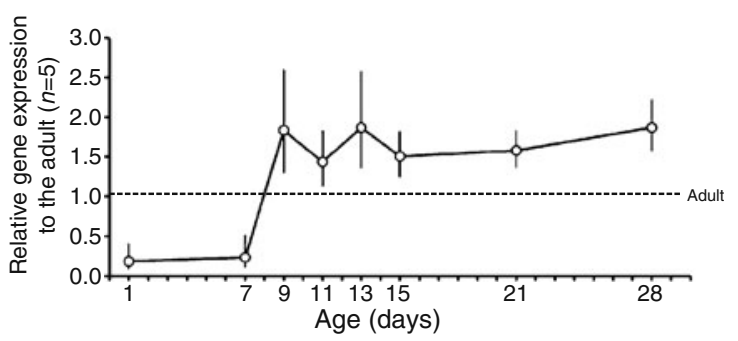

(white circle), carnitine palmitoyltransferase 2 (black triangle); (d) pyruvate kinase (Pklr isoform) (white circle). All but Cptl are expressed at $20 \%$ or less than adult levels during the first week. Data expressed relative to pooled isolated adult islets $(n=5)$. Student's $t$ test for comparison of expression between weeks. ${ }^{*} p<0.05,{ }^{*} p<0.01$, $* * * p<0.001$. ANOVA for repeated measures throughout the time course, $p<0.001$ in all comparisons, except for $P k$

GSIS in vitro [42]. Considering the immaturity of other mitochondrial shuttles and the scarcity of pyruvate-cyclingbased coupling factors, abundant lipids in fetal and neonatal beta cells might act as important coupling factors regulating insulin secretion.

Dietary factors markedly differ during the neonatal period and adults [43], so the lack of glucose-responsive insulin secretion is not really an impairment but an evolutionary adaptation to neonatal fuel. In neonates NEFA and glycerol levels are higher and only after weaning decrease to the adult fed values [44]. In addition, there is the switch from solute carrier family 27 (fatty acid transporter), member 5 (Slc27a5) to member 1 (Slc27a1) fatty acid transporters. Slc27a1 is a major insulin-sensitive long-chain fatty acid transporter, whereas Slc27a5 expression is exquisitely liver-specific in adulthood and its overexpression in other mammalian cells mediates longchain fatty acid uptake [45]. We speculate that since neonatal beta cells lack the major fatty acid transporter SLC27A1, they use the transiently produced SLC27A5 transporter for lipid uptake. Blood glucose levels rise after birth and by P7 are similar to the adult fed state [44]. While these physiological variables may drive the expression of some of the beta cell-specific metabolic enzymes, the different patterns of gene expression that we found suggest complex and varied regulatory networks. The coincidence 
of generalised low expression of many key beta cell genes in neonatal islets and the lack of glucose responsiveness suggests the existence of a critical regulator of functional maturity. Not all key beta cell transcription factors were represented on the microarrays used so determination of such a maturation factor by microarray was not possible. In subsequent experiments [34], we explored the role of Mafa and $P d x 1$ in neonatal beta cell maturation and established that Mafa contributes to the acquisition of glucose responsiveness in neonatal beta cells.

In summary, expression of key metabolic genes including mitochondrial membrane shuttles (malate dehydrogenase, glycerol-3-phosphate dehydrogenase, glutamate oxalacetate transaminase, malic enzyme), pyruvate carboxylase and carnitine palmitoyl transferase 2 were found to be lower in the neonatal islets with increased mRNA expression over the first 4 weeks after birth. Thus, in contrast to previous studies focused on numerous individual genes [18, 19, 37], our results suggest an overall immaturity of the metabolic pathways involved in insulin secretion during the neonatal period.

Acknowledgements The authors thank J. Lock (Joslin Diabetes Center) for her expert technical assistance, J. Schroeder and I-H. Wu (Joslin Diabetes Center) for help with bioinformatics. This study was supported by National Institutes of Health Grants R01 DK 66056 (S. Bonner-Weir), DK $61251 \beta$-cell Biology Consortium (D. Sgroi), the Advanced Microscopy, Genomics and Bioinformatics cores of Joslin Diabetes and Endocrinology Research Center (P30 DK 36836) as well as the Juvenile Diabetes Research Foundation (JDRF 1-2004-120 to S. Bonner-Weir), Diabetes Research and Wellness Foundation, the Graetz Fund, and an important group of private donors. A. Jermendy was supported partially by the Hungarian-American Enterprise Scholarship Fund and TÁMOP-4.2.2-08/01/KMR-2008-2004 research grant, A. Koh by a Singapore National Medical Research Council Research fellowship grant, and T. Aye by a Lawson-Wilkins Fellowship and an Endocrine Fellow Foundation award. The data discussed in this publication have been deposited in NCBI Gene Expression Omnibus [46] and are accessible through GEO Series accession number GSE24790 (www.ncbi.nlm.nih.gov/geo/query/acc. cgi?acc $=$ GSE24790).

Duality of interest The authors declare that there is no duality of interest associated with this manuscript.

\section{References}

1. Nolan CJ, Prentki M (2008) The islet beta-cell: fuel responsive and vulnerable. Trends Endocrinol Metab 19:285-291

2. Schuit F, Flamez D, de Vos A, Pipeleers D (2002) Glucoseregulated gene expression maintaining the glucose-responsive state of beta-cells. Diabetes 51(Suppl 3):S326-S332

3. MacDonald MJ (1993) Estimates of glycolysis, pyruvate (de) carboxylation, pentose phosphate pathway, and methyl succinate metabolism in incapacitated pancreatic islets. Arch Biochem Biophys 305:205-214

4. Ishihara H, Wang H, Drewes LR, Wollheim CB (1999) Overexpression of monocarboxylate transporter and lactate dehydro- genase alters insulin secretory responses to pyruvate and lactate in beta cells. J Clin Invest 104:1621-1629

5. Farfari S, Schulz V, Corkey B, Prentki M (2000) Glucoseregulated anaplerosis and cataplerosis in pancreatic beta-cells: possible implication of a pyruvate/citrate shuttle in insulin secretion. Diabetes 49:718-726

6. MacDonald MJ (1995) Feasibility of a mitochondrial pyruvate malate shuttle in pancreatic islets. Further implication of cytosolic NADPH in insulin secretion. J Biol Chem 270:20051-20058

7. Henquin JC (2009) Regulation of insulin secretion: a matter of phase control and amplitude modulation. Diabetologia 52:739751

8. Grasso S, Messina A, Saporito N, Reitano G (1968) Serum-insulin response to glucose and aminoacids in the premature infant. Lancet 2:755-756

9. Obenshain SS, Adam PA, King KC et al (1970) Human fetal insulin response to sustained maternal hyperglycemia. $\mathrm{N}$ Engl $\mathrm{J}$ Med 283:566-570

10. Asplund K, Westman S, Hellerstrom C (1969) Glucose stimulation of insulin secretion from the isolated pancreas of foetal and newborn rats. Diabetologia 5:260-262

11. Lavine RL, Chick WL, Like AA, Makdisi TW (1971) Glucose tolerance and insulin secretion in neonatal and adult mice. Diabetes 20:134-139

12. Hole RL, Pian-Smith MC, Sharp GW (1988) Development of the biphasic response to glucose in fetal and neonatal rat pancreas. Am J Physiol 254:E167-E174

13. Asplund K (1973) Effects of glucose on insulin biosynthesis in foetal and newborn rats. Horm Metab Res 5:410-415

14. Freinkel N, Lewis NJ, Johnson R, Swenne I, Bone A, Hellerstrom C (1984) Differential effects of age vs glycemic stimulation on the maturation of insulin stimulus-secretion coupling during culture of fetal rat islets. Diabetes 33:1028-1038

15. Hellerstrom C, Swenne I (1991) Functional maturation and proliferation of fetal pancreatic beta-cells. Diabetes 40(Suppl 2):89-93

16. Grill V, Lake W, Freinkel N (1981) Generalized diminution in the response to nutrients as insulin-releasing agents during the early neonatal period in the rat. Diabetes 30:56-63

17. Bliss CR, Sharp GW (1992) Glucose-induced insulin release in islets of young rats: time-dependent potentiation and effects of 2bromostearate. Am J Physiol 263:E890-E896

18. Rorsman P, Arkhammar P, Bokvist K et al (1989) Failure of glucose to elicit a normal secretory response in fetal pancreatic beta cells results from glucose insensitivity of the ATP-regulated $\mathrm{K}^{+}$channels. Proc Natl Acad Sci USA 86:4505-4509

19. Tan C, Tuch BE, Tu J, Brown SA (2002) Role of NADH shuttles in glucose-induced insulin secretion from fetal beta-cells. Diabetes 51:2989-2996

20. Gotoh M, Maki T, Satomi S et al (1987) Reproducible high yield of rat islets by stationary in vitro digestion following pancreatic ductal or portal venous collagenase injection. Transplantation 43:725-730

21. Sgroi DC, Teng S, Robinson G, LeVangie R, Hudson JR Jr, Elkahloun AG (1999) In vivo gene expression profile analysis of human breast cancer progression. Cancer Res 59:5656-5661

22. Emmert-Buck MR, Bonner RF, Smith PD et al (1996) Laser capture microdissection. Science 274:998-1001

23. Ahn YB, Xu G, Marselli L et al (2007) Changes in gene expression in beta cells after islet isolation and transplantation using laser-capture microdissection. Diabetologia 50:334-342

24. Luo L, Salunga RC, Guo H et al (1999) Gene expression profiles of laser-captured adjacent neuronal subtypes. Nat Med $5: 117-122$

25. da Huang W, Sherman BT, Lempicki RA (2009) Systematic and integrative analysis of large gene lists using DAVID bioinformatics resources. Nat Protoc 4:44-57 
26. Dennis G Jr, Sherman BT, Hosack DA et al (2003) DAVID: Database for Annotation, Visualization, and Integrated Discovery. Genome Biol 4:P3

27. Huggett J, Dheda K, Bustin S, Zumla A (2005) Real-time RT-PCR normalisation; strategies and considerations. Genes Immun 6:279284

28. Applied Biosystems (2001) User Bulletin \#2 ABI Prism 7700 Sequence Detection System

29. Newgard CB, McGarry JD (1995) Metabolic coupling factors in pancreatic beta-cell signal transduction. Annu Rev Biochem 64:689-719

30. Quintens R, Hendrickx N, Lemaire K, Schuit F (2008) Why expression of some genes is disallowed in beta-cells. Biochem Soc Trans 36:300-305

31. Yaney GC, Schultz V, Cunningham BA, Dunaway GA, Corkey BE, Tornheim K (1995) Phosphofructokinase isozymes in pancreatic islets and clonal beta-cells (INS-1). Diabetes 44:1285-1289

32. Guay C, Madiraju SR, Aumais A, Joly E, Prentki M (2007) A role for ATP-citrate lyase, malic enzyme, and pyruvate/citrate cycling in glucose-induced insulin secretion. J Biol Chem 282:3565735665

33. Lu D, Mulder H, Zhao P et al (2002) ${ }^{13} \mathrm{C}$ NMR isotopomer analysis reveals a connection between pyruvate cycling and glucose-stimulated insulin secretion (GSIS). Proc Natl Acad Sci USA 99:2708-2713

34. Aguayo-Mazzucato C, Koh A, El Khattabi I, Li W-C, Toschi E, Jermendy A, Juhl K, Mao K, Weir GC, Sharma A, Bonner-Weir S (2011) MafA and Pdx1 contribute to acquisition of glucoseresponsive insulin secretion in neonatal rat $\beta$-cells. Diabetologia. doi:10.1007/s00125-010-2026-z

35. Brun T, Roche E, Assimacopoulos-Jeannet F, Corkey BE, Kim KH, Prentki M (1996) Evidence for an anaplerotic/malonyl-CoA pathway in pancreatic beta-cell nutrient signaling. Diabetes 45:190-198
36. Antinozzi PA, Ishihara H, Newgard CB, Wollheim CB (2002) Mitochondrial metabolism sets the maximal limit of fuel-stimulated insulin secretion in a model pancreatic beta cell: a survey of four fuel secretagogues. J Biol Chem 277:11746-11755

37. Hasan NM, Longacre MJ, Stoker SW et al (2008) Impaired anaplerosis and insulin secretion in insulinoma cells caused by small interfering RNA-mediated suppression of pyruvate carboxylase. J Biol Chem 283:28048-28059

38. Jensen MV, Joseph JW, Ilkayeva O et al (2006) Compensatory responses to pyruvate carboxylase suppression in islet beta-cells. Preservation of glucose-stimulated insulin secretion. J Biol Chem 281:22342-22351

39. Jitrapakdee S, Wutthisathapornchai A, Wallace JC, MacDonald MJ (2010) Regulation of insulin secretion: role of mitochondrial signalling. Diabetologia 53:1019-1032

40. Thorrez L, Laudadio I, Van Deun K et al (2011) Tissue-specific disallowance of housekeeping genes: the other face of cell differentiation. Genome Res 21:95-105

41. Eto K, Tsubamoto Y, TerAUChi Y et al (1999) Role of NADH shuttle system in glucose-induced activation of mitochondrial metabolism and insulin secretion. Science 283:981-985

42. Rubi B, Antinozzi PA, Herrero L et al (2002) Adenovirusmediated overexpression of liver carnitine palmitoyltransferase I in INS1E cells: effects on cell metabolism and insulin secretion. Biochem J 364:219-226

43. Page MA, Krebs HA, Williamson DH (1971) Activities of enzymes of ketone-body utilization in brain and other tissues of suckling rats. Biochem J 121:49-53

44. Henning SJ (1981) Postnatal development: coordination of feeding, digestion, and metabolism. Am J Physiol 241:G199-G214

45. Stahl A (2004) A current review of fatty acid transport proteins (SLC27). Pflugers Arch 447:722-727

46. Barrett T, Troup DB, Wilhite SE et al (2009) NCBI GEO: archive for high-throughput functional genomic data. Nucleic Acids Res 37:D5-D15 